\title{
La Mettrie e Mandeville: il nesso tra morale e politica e il ruolo delle virtù
}

\section{Giulio Panizza}

\section{(2) OpenEdition}

1 Journals

\section{Edizione digitale}

URL: http://journals.openedition.org/studifrancesi/3597

DOI: 10.4000/studifrancesi.3597

ISSN: 2421-5856

\section{Editore}

Rosenberg \& Sellier

\section{Edizione cartacea}

Data di pubblicazione: 1 décembre 2012

Paginazione: 414-421

ISSN: 0039-2944

\section{Notizia bibliografica digitale}

Giulio Panizza, «La Mettrie e Mandeville: il nesso tra morale e politica e il ruolo delle virtù», Studi Francesi [Online], 168 (LVI | III) | 2012, online dal 30 novembre 2015, consultato il 06 mars 2021. URL: http://journals.openedition.org/studifrancesi/3597 ; DOI: https://doi.org/10.4000/studifrancesi.3597

\section{(c) (†) $\odot$}

Studi Francesi è distribuita con Licenza Creative Commons Attribuzione - Non commerciale - Non opere derivate 4.0 Internazionale. 


\section{La Mettrie e Mandeville: il nesso tra morale e politica e il ruolo delle virtù}

Il rapporto tra morale e politica, sia in La Mettrie che in Mandeville, con una analogia sorprendente per il modo in cui si dice, viene rovesciato rispetto alla tradizione antica e classica: lungi dall'avere a che fare con l'aristotelico ethos della polis, quella relazione è intesa come ruolo di comando della politica - con i suoi potenti apparati ideologici e di potere - sulla morale. La struttura espositiva e lo schema concettuale che La Mettrie impiega sono pressoché identici, come s'è detto, anche nell'espressione lessicale usata - fatta salva ovviamente la differenze delle lingue - a quelli esibiti da Mandeville nell'Enquiry into the Origin of Moral Virtue. «Le virtù morali», scrive infatti Mandeville, «sono la prole politica che l'adulazione ha concorso a generare dall'orgoglio» ${ }^{1}$. «La morale», osserva La Mettrie nel Discours préliminaire, «trae vita dalla politica e non è per nulla opera della natura o della filosofia o della ragione, che sono tutti sinonimi» ${ }^{2}$. Sul significato di questa intensa somiglianza, che ha una chiara ascendenza hobbesiana e che non è sorretta da una reciproca diretta conoscenza critica, ossia sulla configurazione dell'elenco e della funzione delle virtù che implica e sulla loro influenza, insieme alla distinta configurazione delle manovre concettuali predisposte - più raffinata e complessa quella di Mandeville, più tagliente e diretta quella di La Mettrie - intendo argomentare.

La tesi sul relativismo etico, in relazione alle virtù, è affermata con forza da La Mettrie già nell'Anti-Sénèque: «Le virtù sono relative alla società, di cui formano al tempo stesso l'ornamento e il sostegno» ${ }^{3}$. Si tratta della conclusione di un ragionamento che si basa su tre passaggi fondamentali. Il primo è costituito dalla rottura del nesso tra virtù e felicità, così strettamente legate nell'Etica Nicomachea e nella precettistica morale del tempo, e dalla difesa del carattere fisico del piacere o, come è detto ne L'Homme machine, della «felicità corporea». Lo stesso fortuito coordinamento vitale che agisce nella circolazione, il medesimo gioco di solidi e liquidi che produce il genio immenso o lo spirito limitato, non sono altro che l'effetto di quella sensibilità che «ci rende felici o infelici, poiché la felicità non ha altra origine, come ci insegna l'uniformità dell'ordine naturale» ${ }^{4}$. Qui La Mettrie incontra Epicuro, e le sue nozioni di aponia, il controllo e l'equilibrio stabile dei piaceri, e di atarassia, l'assenza di turbamento interiore che ne deriva, mentre respinge in modo polemico l'apatia del sag-

(1) Mandeville, The Fable of the Bees, 1729 (tr. it. introduzione e cura di T. MAGRI, La favola delle api, Roma-Bari, Laterza, 1997, p. 30). Sull'opera di Mandeville sono da vedere: M.-M. Goldsmith, Private vices, Public benefits. B. Mandeville, Cambridge, Cambridge UP, 1985; M.-E. SCRIBANO, Natura umana e società competitiva. Studio su Mandeville, Feltrinelli, Milano, 1980; I. Primer, Mandeville and Shaftesbury, in Mandeville Studies, The Hague, M. Nijhof, 1975, pp. 126-148.

(2) La Metrrie, Discours préliminaire, 1751 (tr. it. introduzione e cura di S. Moravia, Opere filo- sofiche, Roma-Bari, Laterza, 1974, p. 6). Sull'opera di La Mettrie sono da vedere, oltre all'importante introduzione di Moravia: A. Thomson, La Mettrie's Discours préliminaire, Genève, Droz, 1981; J. FALVEY, L'anarchisme de La Mettrie, in «Corpus», n. 5/6, 1987; K. Wellman, La Mettrie, London, Duke UP, 1992; C. Morilhat, La Mettrie. Un matérialisme radical, Paris, PUF, 1997.

(3) La MetTrie Anti-Sénéque, 1750 (tr. it. in Opere filosofiche, cit., p. 319).

(4) Ivi, p. 307. 
gio stoico, l'autonomia razionale o l'impassibilità del sapiente, fondata sulla rinuncia alle passioni, da cui sono invece dominati gli stolti, i fauloi: «Come ci sentiamo antistoici!», scrive nell'Anti-Sénèque, «Questi filosofi sono severi, tristi, duri; noi saremo dolci, allegri, compiacenti. Tutti anima, essi prescindono dal loro corpo; tutti corpo, noi prescinderemo dalla nostra anima ${ }^{5}$. È su questa base che, mediante la misurazione comparativa dei piaceri suggerita da Epicuro, si è condotti alla «contentezza dello spirito». Del resto, il termine volupté indica presso i philosophes non tanto o non solo l'accentazione fisica del piacere - sebbene La Mettrie non la disdegni in alcune passi estremi della sua opera: «E anche tu voluttuoso pensa solo al tuo corpo... che la polluzione e il godimento, lubrichi rivali, si succedano a turno e ti facciano sciogliere per il piacere ${ }^{6}-$ quanto una felicità più sottile, connessa alla consapevolezza che potenzia il desiderio e conduce a uno stato di perfetto appagamento e deliziosa immobilità. Nel suo Agathon Le Grec osserva infatti come la volupté consista nel gout de l'esprit e nella réflexion. E Saint-Hyacinthe nelle Recherches philosophiques nota: «Quand je réfléchis sur ce que j’appelle Bonheur, il me semble que c'est un sentiment d'approbation de l'état où je suis, d'où nait ce que j'appelle constantement joie, tranquillité de l'âme»'.

È la democratizzazione del bonbeur la seconda mossa concettuale che giustifica il relativismo etico di La Mettrie, ossia la dipendenza del moral dal physique e pertanto il ruolo essenziale della conformazione psico-fisica - lo scorrere degli "spiriti animali" dal cervello ai nervi fino agli organi di senso - nello spiegare la differente capacità o attitudine al piacere fra gli uomini. Da questo punto di vista, legato all'organisation, si riconoscerà che «la felicità è possibile per i dotti e per gli ignoranti, per i ricchi e per i poveri, insomma per tutti $\mathrm{i}$ ceti e quel che più conta, ciò che farà rivoltare gli spiriti prevenuti e bigotti, per i buoni come per i malvagi»». Sulla connotazione dura e individuale della felicità, connessa a una gradevole stimolazione corporea di per sé neutra e avalutativa, La Mettrie costruisce il suo amoralismo descrittivo: «Non mi si dica che il mio è un invito al delitto, giacché io invito soltanto alla tranquillità nel delitto; in generale l'uomo sembra seguire più la foga del sangue e delle sue passioni che non le idee edificanti ricevute fin dall'infanzia. Non sostengo affatto, Dio non voglia, la malvagità, troppo contraria al mio carattere; piuttosto la compatisco, perché ne trovo la fonte nell'uomo che qualche volta è difficile e perfino impossibile da domare»". E in modo ancor più eloquente nel Système d'Epicure: «Siamo nelle mani della natura come una pendola è in quelle di un orologiaio, essa ci ha plasmato come ha voluto; insomma, seguendo l'impressione dei movimenti primitivi che ci governano, non siamo più criminali di quanto non lo sia il Nilo quando compie le sue inondazioni e il mare quando s'infuria». Non solo la propensione alla felicità è indiscriminata e non dipende dai meriti o dai talenti personali, ma neppure è possibile identificarla con il possesso di una inclinazione alla benevolenza, alla compassione o alla simpatia verso i nostri simili, come sostenevano le dottrine del moral sense di Hutcheson o della risonanza emotiva di Hume. Vi è dunque per l'autore de L'Homme machine una inabilità costitutiva al bene, incardinata nel complesso motivazionale, moralmente non imputabile.

Separata dalla felicità, resa priva di un contenuto autonomo, la virtù - e siamo al terzo movimento dell'etica di La Mettrie - diviene artificio, simulazione, rito sociale: «Ammettete in buona fede», ci ricorda il Discours, «che giusto è solo colui che pesa la

(5) Ivi, p. 302

(6) Ivi, p. 357 .

(7) Cit. in R. MauzI, L'idée de Bonbeur au XVIII siècle, Paris, Colin, 1967, p. 125.

(8) La MetTrie, Anti-Sénèque (tr. it. cit., p. 305).

(9) Ivi, p. 287. 
giustizia con la bilancia della società» ${ }^{10}$. La Mettrie distingue fra esibizione pubblica, in cui ogni ceto rappresenta le sue virtù, e simulazione individuale di buoni sentimenti o "egoismo mascherato". Così, secondo un elenco che sembra ripreso pari pari dal Mandeville di The Fable of the Bees, mostra come il cittadino zelante e bisognoso di favori formuli voti per la sua patria, l'ufficiale coraggioso e alla ricerca di una promozione guidi il soldato ad essere intrepido, il moralista di buon senso fornisca precetti accettabili, lo storico di corte elenchi i grandi esempi da imitare nell'antichità, il medico eserciti con laute prebende la sua arte di conservare gli uomini. Mentre il singolo, sull'altro versante della scena colto nel suo momento aurorale, appresta la sua recita virtuosa sul palcoscenico del mondo guidato dalla ricerca dell'onore, dalla forza della vanità, dal timore della vergogna e dalla frusta dei rimorsi. Sono i quietivi della volontà, per dar corso a un'espressione schopenhaueriana, che l'educazione e il potere impiegano per controllare e orientare gli uomini. «La virtù», afferma La Mettrie con una sintesi volutamente sgradevole, «è una brutta vecchia, che si ricerca per il lustro che pende dalle sue orecchie o per il denaro che fa guadagnare» ${ }^{11}$. E in modo più analitico:

Possiamo dire delle virtù ciò che Zenone diceva dei vizi, cioè che sono tutti uguali. Ma l'onore e la gloria, fantasmi seducenti, hanno ricevuto un nome per servire da seguito alla virtù che essi stimolano. Il disprezzo, l'ignominia, i rimorsi sono stati invece collegati ai vizi per seguirli, spaventarli, servire loro da Erinni. Insomma si è voluto scuotere l'immaginazione degli uomini, sfruttando con questo la loro sensibilità, onde ciò che in sé è solo una chimera, divenisse nelle relazioni umane un bene reale ${ }^{12}$.

La necessità dei legami sociali, e delle loro articolazioni gerarchiche - lo Stato come "progetto del ricco" dirà Rousseau nel Discours sur l'inégalité - ha dunque implicato la nascita delle nozioni di vizio e di virtù, la cui origine è quindi di natura politica, ossia pertiene alla configurazione di invasivi sistemi ideologici che incanalano l'adesione al patto civile dato. «Per chi è costruito il mirabile apparato della politica?», si chiede La Mettrie in un passo del Système d'Epicure in cui affiora l'elitismo caratteristico della tradizione libertina, «per uomini che altrimenti non avrebbero avuto altri freni adeguati - una specie che purtroppo costituisce il maggior numero degli individui - una specie imbecille, bassa, strisciante, da cui la società ha creduto di poter trarre qualche vantaggio solo catturandola con l'interesse, primo motore di tutti gli uomini, creatore di una felicità chimerica» ${ }^{13}$.

La lettura, fra gli altri, del manoscritto clandestino L'Examen de la religion, in cui la fede cristiana è descritta come "favola immaginaria" utile per tenere a freno i popoli in nome dell'alleanza fra trono e altare, consente infine a La Mettrie di mettere a punto, correggendo parzialmente l'argomentazione de L'Homme machine, una precisa e potente teoria in grado di mostrare nella condotta dell'uomo l'efficacia della distinzione tra vergogna, o autorità morale esterna, e rimorso, o autorità morale interna. Se la vergogna, sorretta dai riti condivisi, ci conduce a porre l'opinione del mondo al centro del nostro agire - così rinunciando ad ogni autenticità - il rimorso, questo "affliggente pregiudizio", creando una continua tensione interiore ci allontana da quel sentimento di piacere in cui consiste la dolcezza del vivere - la "rosa dell'esistenza" - e ci allena alla sottomissione, alla tristezza, al rispetto passivo dell'autorità. E tuttavia La Mettrie sottolinea come l'efficacia di questi condizionamenti artificiali

(10) La Mettrie, Discours préliminaire (tr. it. cit., p. 20).

(11) La MetTrie, Anti-Sénèque (tr. it. cit., p. 320).
(12) Ivi, p. 318.

(13) La MetTrie, Système d'Epicure, 1751 (tr. it. cit., p. 290). 
non sia assoluta. Essi si scontrano con il modo in cui l'uomo è fatto, contraddicono la sua costituzione psico-fisica, sulla quale insistono allo stesso modo del badile contro la roccia: «il filosofo, troppo illuminato per considerarsi colpevole di pensieri e azioni che nascono e si formano suo malgrado, sospirando sulla funesta condizione umana, non si lascia rodere da quei carnefici che sono i rimorsi, frutti amari dell'educazione che non sono mai stati appesi all'albero della natura $>^{14}$. È ben vero, come ci insegna il sensismo di Condillac, che il meccanismo associativo della vita psichica, l'abitudine, generata dalla sensazione ripetuta, è assai influente e costituisce una notevole arma, emancipativa o tirannica, per l'indirizzo del comportamento. Ma non è tutto. La dialettica fra il timore dell'opinione altrui e l'auto-accusa della coscienza si stempera non appena si rifletta sulla base sensibile della felicità. Gli è che l'analisi di La Mettrie riposa, e forse in questo consiste la sua originalità maggiore nel panorama del materialismo illuministico francese - ciò che lo rese "scandaloso" per i suoi contemporanei - su di una forma di soggettivismo radicale connesso non semplicemente alla preferenza di sé hobbesiana o all'amour propre roussauiano, bensì alla nozione di organisation. Su di essa l'attitudine plasmante della società, l'indicazione del bene comune, che carica gli uomini "come un orologio" inculcando loro le illusioni di una morale universalistica, sa operare solo per un breve tratto. Dunque La Mettrie solleva il velo del nesso tra potere-sapere-condotta, ma nel momento in cui lo fa non prende troppo sul serio la forza di quel velo, che è ovviamente altra cosa dalla descrizione del suo agire. Come scrive nel Discours, in un contesto nel quale è forte l'intento polemico: «Io ho creduto di provare che l'uomo è una macchina governata imperiosamente da un fatalismo assoluto» ${ }^{15}$.

È in Mandeville che l'idea della artificialità della morale, ossia il suo carattere di finzione e il suo possibile uso manipolatorio, raggiunge le punte estreme. Non solo e non tanto nel celebre paradosso di The Fable of the Bees - "vizi privati, pubblici benefici" - o nell'affermazione, contenuta in A Search into the Nature of Society (nell'edizione del 1723 di The Fable) che solo dal male, naturale e morale, il bene, ovvero dal lusso l'incremento delle arti e delle industrie e la conseguente crescita del benessere generale, quanto nella definizione dei termini che spiegano e muovono questo processo: vizio e virtù, onore e vergogna. Il punto iniziale dell'argomentazione di Mandeville - in cui si riflette la discussione a lui coeva intorno al conflitto tra virtù e commercio - è come noto la critica del concetto rigoristico di virtù, basato sulla abnegazione e sulla mortificazione degli impulsi naturali, cui è opposta la molteplicità dei bisogni e la varietà degli scambi che danno vita a una società prospera: «la semplice virtù non può far vivere le nazioni nello splendore; chi vuole fare tornare l'età dell'oro deve tenersi pronto per le ghiande come per l'onestà» ${ }^{16}$. Si tratta, in termini più tecnici, di un elogio del ruolo dinamico del mercato allo stato nascente: «maggiore è la varietà dei bisogni, più grande è il numero di individui che possono trovare il loro interesse privato nel lavorare per il bene degli altri, e uniti insieme formare un solo corpo». Tuttavia, seguire e soddisfare i propri interessi personali non basta, perché rappresenta il movente e non il fine del corpo sociale; altrimenti è la guerra di tutti contro tutti per il possesso dei beni, lo stato di natura ferino a cui pone termine il Leviatano di Hobbes. La complessità della convivenza concorrenziale richiede norme, regole, divieti. Il monopolio della forza, assicurato dallo Stato, trova qui il suo luogo d'esercizio. Eppure, osserva Mandeville, nemmeno la forza è sufficiente: «non è possibile con la sola forza rendere l'uomo docile e fargli compiere i progressi di cui è capace» ${ }^{17}$. Sono le relazioni di potere e le loro strutture di influenza che mo-

(14) Ivi, p. 279.

(15) La MetTrie, Discours préliminaire (tr. it. cit., p. 17).
(16) Mandeville, The Fable of the Bees (tr. it. cit., p. 21).

(17) Ivi, p. 17. 
dellano il nostro modo di pensare e di sentire, interiorizzando, nelle forme date, le nozioni di virtù e di vizio. In un passo centrale della sua opera Mandeville illustra il tema. Legislatori, saggi e politici hanno diviso la società in due classi: quella di coloro che vengono considerati incapaci di frenare i propri istinti individuali - «persone abbiette e di animo vile, sempre a caccia di godimenti immediati» - e quella di coloro che vengono ritratti come dotati delle più alte qualità spirituali - «nobili creature di animo elevato, libere dal sordido egoismo». Quest'ultima, nel racconto di sfondo che non esita a usare toni elegiaci e a far ricorso ad ogni pathos supererogatorio, è la classe di coloro che detengono il comando, mentre la prima costituisce la classe dei sudditi. Ora, i "reggitori della cosa pubblica", cristallizzando la funzione sociale di dominio in una norma etica, chiamano «virtù ogni azione con cui l'uomo, andando contro l'impulso della natura, ricercasse il vantaggio degli altri o la vittoria sulle proprie passioni per l'ambizione razionale di essere buono, e danno il nome di vizio a tutto ciò che, senza nessun riguardo per il bene collettivo, l'uomo facesse per soddisfare un suo appetito» ${ }^{18}$. In realtà la rappresentazione a cui assistiamo, osserva Mandeville, è una proiezione mitica e rovesciata nel cielo della ideologia, la quale descrive un modo di condursi che non esiste mai - la virtù come autonomia razionale del ceto dominante e il vizio come signorìa dell'impulso presso le classi subalterne - e che ciononostante si fa accettare quale narrazione obbiettiva. Come avviene questo miracolo, per così dire, tale da rendere gli uomini docili e maneggevoli e da permettere ad alcuni di raccogliere i frutti della fatica e della rinuncia di altri? A questo punto l'analisi di Mandeville si affianca a quella di La Mettrie e procede per il medesimo sentiero argomentativo. Mostra pertanto come, attraverso "esempi mirabili" e considerando che nessuno «era così selvaggio da non essere incantato dalla lode o così spregevole da sopportare con pazienza il disprezzo», moralisti e filosofi incominciarono a istruire gli uomini con le nozioni di onore e di vergogna. Anzi, ad essere più precisi, le nozioni di onore e di infamia vennero manovrate per muovere le passioni dell'orgoglio e della vergogna. Della vergogna, quale forma introiettata del giudizio sociale sul comportamento, Mandeville scrive: «durante tutta l'educazione cerchiamo di accrescere, anziché diminuire o eliminare, il senso di vergogna; e l'unico rimedio prescritto per esso è la stretta osservanza di determinate regole, in modo da evitare le cose che possono suscitare in noi questo penoso sentimento; ma quanto a eliminarlo o a curare l'uomo da esso, il politico preferirebbe ucciderlo» ${ }^{19}$. L'adulazione dell'orgoglio, tramite il rilucente simulacro dell'onore, è il secondo potente strumento che spinge gli uomini a credere nelle propria natura eccellente: «Se l'adulazione era il movente più forte che si potesse usare nei confronti degli uomini, parimenti l'orgoglio era a tal punto inseparabile dalla loro stessa essenza che, privato di esso, il composto di cui sono fatti mancherebbe di uno degli ingredienti principali. Considerato tutto questo è difficile dubitare che lezioni e rimproveri dovessero indurre molti, specialmente i più fieri, $\mathrm{i}$ più risoluti, i migliori fra loro, a sopportare mille disagi e ad affrontare mille difficoltà per avere il piacere di annoverarsi tra i membri della classe superiore, quella che non mira ad altro cha al benessere pubblico e alla vittoria sulle proprie passioni $\rangle^{20}$.

Nella dottrina di Mandeville, allo stesso modo che in La Mettrie, non ha dunque campo - ne è infatti il simmetrico antagonista - la teoria del sentimento morale di Shaftesbury. La virtù non obbedisce ad una primaria inclinazione di benevolenza del cuore umano o ad un moral sense che si risveglia nel perseguimento del pubblico interesse e in cui si riflettono l'armonia e l'unità dell'universo. La "gentile" traccia delle Characteristics, quando si mette alla prova del mondo concreto, conduce suo 
malgrado, proprio perché è contraria alla natura dell'uomo come questa appare, all'ipocrisia e all'autoinganno: «L'idea, del tutto priva di fondamento, che gli uomini possano essere virtuosi senza sforzo apre il varco alla menzogna e nello stesso tempo ci rende sconosciuti a noi stessi» ${ }^{21}$. Il difetto maggiore di ogni concezione finora sostenuta della virtù, afferma Mandeville, sia nella accezione rigoristica della tradizione classica, sia nella accezione fluida e diretta della morale del sentimento, è dato dal fatto che entrambe ne ignorano il tratto essenziale, ossia che sempre la virtù rappresenta la recita una prospettiva di dominio sociale ed è funzionale all'ordinato ed efficace svolgersi del processo di civilizzazione: «I primi elementi della moralità, introdotti da abili politici per rendere gli uomini utili gli uni agli altri, furono inventati soprattutto affinché l'ambizioso potesse ricavare il maggiore beneficio e governare grandi numeri di uomini con facilità e sicurezza» ${ }^{22}$. Se la virtù è mascheramento della "predilezione per se stessi" (Self-liking), come Mandeville non ha dubbio, ogni ricerca di un fondamento universale mente sulle vere ragioni dell'agire umano.

La comparazione del carattere discendente della relazione tra politica e morale, in La Mettrie e in Mandeville, richiede ora alcune precisazioni in merito a similitudini e differenze. I termini impiegati, s'è detto, sono spesso pressoché identici, così come lo schema interpretativo che viene proposto. Pessimismo antropologico («senza vizi la superiorità della specie umana non si sarebbe mai manifestata» $)^{23}$; descrittivismo eticopolitico («se l'uomo fosse stato per natura umile e indifferente all'adulazione il politico non avrebbe mai potuto raggiungere i propri fini» $)^{24}$; genealogia del rapporto tra potere, sapere e istituzioni («i rimorsi sono strumentali perché si connettono a un uso improprio della religione, illusione coltivata per intimidire i popoli e garantire la sicurezza del governo») $)^{25}$, vanno di pari passo e collimano nei due autori. E tuttavia l'opera di demistificazione delle costruzioni ideali, falsamente neutre e paternalistiche, con cui le società giustificano se stesse e le loro interne articolazioni di diseguaglianza, si arresta in La Mettrie alla indagine sul ruolo della nostra soggettività in questo processo e lo fa coincidere, in ultima analisi, con il sentimento immediato dell'esistenza e con la pratica dello tolleranza: «Siamo in questo mondo per cercare e godere i piaceri della vita; poiché il caso ci ha gettato in essa il nostro primo e più ragionevole obiettivo sia quello di vivere tranquilli, in modo comodo e sereno» ${ }^{26}$. Diversamente in Mandeville, con mossa più sottile da scienziato della politica, la ricerca si situa sui requisiti delle istituzioni che, sia pure contraddittoriamente, condizionano le nostre pratiche, sicché si deve ritenere che i vizi privati, attraverso l'accorta amministrazione di un abile politico, possono diventare pubblici benefici ${ }^{27}$. La "scuola del sospetto", per usare un'espressione di Nietzsche ripresa da Paul Ricoeur, ci conduce con La Mettrie allo zoccolo duro del determinismo psico-fisico e alla felicità corporea, oltre cui non si può procedere; con Mandeville ci indica le vie sinuose, aggrovigliate, per niente lineari né tranquillizzanti del processo di civilizzazione. La virtù è certo artificio. Peggio, è strumento di controllo e di dominio su di un uomo manovrato dall'adulazione dell'orgoglio e dal timore della vergogna, ma insieme è una nozione utile - per quanto irreale - a regolare il conflitto che continuamente rinasce fra gli "egoismi naturali" degli individui. L'analisi di Mandeville coglie e presenta i due versi del ragionamento.

Forse la più influente, meditata e originale rappresentazione di quello che potremmo definire, con un po' di enfasi, il paradigma La Mettrie/Mandeville, applicato alla capacità dei legislatori di unire felicità individuale e felicità collettiva, la possiamo

(21) Ivi, p. 235.

(22) Ivi, p. 28.

(23) Ivi, p. 237.

(24) Ivi, p. 238.
(25) La MetTrie, Anti-Sénèque (tr. it. cit., p. 325).

(26) Ivi, p. 150.

(27) Mandeville, The Fable of the Bees (tr. it. cit., p. 267). 
rintracciare nelle opere di Helvétius. Anche in lui il tono di base rinvia a Hobbes e alla sua negazione di efficacia all'etica aristotelica e scolastica. La teoria del sommo bene appariva infatti all'autore del De Cive una costruzione in gran parte insicura e poggiante, come aveva osservato Descartes a proposito dei trattati di morale degli antichi nel Discours de la méthode, su fango e sabbia. Piacere e utilità sembravano, al contrario, candidati autorevoli a interpretare la condotta dell'uomo perché erano più legati alla sua struttura fisica, in cui avvengono processi suscettibili di analisi quantitativa e dunque esattamente osservabili, processi sui quali la nuova scienza si era esercitata con successo nelle sue brillanti e conformi descrizioni. Quel che si intendeva perseguire non era insomma un ideale da rappresentare ma una spiegazione accurata e immanente nella valutazione di ciò che è bene e di ciò che è male per l'uomo. Accentuando tale curvatura empirica, per così dire, dell'etica Helvétius nel De l'esprit scrive: «Ho creduto opportuno considerare la morale come tutte le altre scienze e costruire una morale come fisica sperimentale» ${ }^{28}$. Su questa base diventa possibile costruire un discorso credibile sulle virtù e avviare una indagine efficace sui moventi della condotta. La ricerca del piacere e la fuga dal dolore costituiscono infatti in natura - nella sfera della soddisfazione dei bisogni primari - le leve principali dell'agire e in essi consiste per l'uomo il bene. Attraverso la nascita della società civile poi, le parole bene e male, «create per esprimere le sensazioni di piacere e di dolore fisico che riceviamo dagli oggetti esterni, saranno estese a tutto ciò che può procurarci l'una o l'altra di queste sensazioni, accrescerle o diminuirle, come la ricchezza e la povertà ${ }^{29}$. Nell'affresco antropologico e narrativo dell'ineguale procedere della civilizzazione dipinto nel De l'bomme, Helvétius sottolinea come le nozioni primitive di piacere e dolore mutino inesorabilmente nome per trasformarsi, nella strutturazione complessa della vita morale e politica, in amor di sé e in desiderio di potere. La straordinaria capacità degli uomini - che li rende diversi da tutti gli altri viventi - di creare organizzazioni sempre più stratificate e diffuse, accentua la potenziale competizione per l'esercizio della egemonia di ciascuno sugli altri e ne cambia la forma; ciò che Hume aveva descritto nel Treatise of Human Nature attraverso la nozione di "circostanze di giustizia", ovvero esaminando il conflitto tra circostanze esterne - relativa penuria delle risorse e relativa instabilità del loro possesso - e circostanze interne - le nostre particolaristiche concezioni della vita buona ${ }^{30}$. Ma come, si domanda Helvétius, questo processo, in apparenza inevitabile, può essere arrestato o composto nei modi corretti e sorvegliati di una convivenza condivisa? $\mathrm{E}$ in che maniera può essere fatta valere la distinzione, di grande importanza per significare il fine della comunità umana, tra virtù di pregiudizio - tali che non contribuiscono in nulla al benessere pubblico, come la castità delle vestali - e virtù vere - tali che favoriscono e espandono la pubblica felicità? Il punto di mediazione, ovviamente, è la reciprocità di riconoscimento tra interesse individuale e interesse generale. La funzione che la promuove è quella del legislatore, ossia la sfera politica: «Tutta l'arte del legislatore», recita un passo decisivo di De l'esprit, «consiste nel forzare gli uomini, facendo centro sul sentimento dell'amor di se stessi, ad essere sempre giusti gli uni verso gli altri. Ora, per istituire simili leggi, è necessario sapere, in primo luogo, che gli uomini non sono nati né buoni né cattivi, ma pronti ad essere l'una o l'altra cosa a seconda che un interesse comune li unisca o li divida; che il sentimento di preferenza che ciascuno prova per sé, sentimento cui è legata la conservazione della specie, è

(28) HeLvetius, De l'esprit, 1758 (tr. it. parziale a cura di A. Postigliola, Dello spirito, Roma, Editori Riuniti, 1976, p. 4.

(29) Ivi, p. 114.
(30) Hume, Treatise on Human Nature, 1739 (tr. it. e introduzione. di E. LeCALDANO, Trattato sulla natura umana, Roma-Bari, Laterza, 1975, vol. II, pp. 504-512). 
scolpito dalla natura in modo incancellabile e che il suo sviluppo ha dato origine alle passioni donde sono usciti tutti i nostri vizi e le nostre virtù» ${ }^{31}$. In tal modo morale e legislazione divengono «un'unica e medesima scienza» e favoriscono la virtù come sintesi, nella pubblica felicità, dell'interesse dei singoli, poiché è solo con buone leggi che «si possono formare uomini virtuosi» ${ }^{32}$.

Il resoconto che Helvétius ci fornisce è a prima vista piano e lineare, ma implica una duplice esigente mossa concettuale: realistico-descrittiva ( $\ll$ il sentimento dell'amor di sé è l'unica base su cui si possono gettare le fondamenta di una morale utile») $)^{33}$ e anti-dogmatica («la natura, come dice Pascal, non è nient'altro che la nostra prima abitudine») $)^{34}$. Evitata la "fallacia patetica", ovvero l'eccesso di prescrittivismo, e ancorata l'analisi della condotta alla osservazione di come l'uomo di fatto è, appare egualmente possibile considerare che la sua natura non è fissa e immutabile, che anzi il processo di civilizzazione è innescato da una perfettibilità - favorita dall'educazione e dalle istituzioni - capace di plasmare l'uomo alla socievolezza. O forse, con una tesi più ardita, ripresa da Pascal e avanzata già da Montaigne nei suoi Essais, che natura umana e costumi coincidono, in uno scambio continuo di livello, dove il costume scende a occupare il posto della natura e la natura si trasforma in costume ${ }^{35}$. Così un abile e attento legislatore, governando questo processo - ecco la lezione di Mandeville - riesce a vincere l'ignoranza che richiude l'uomo in se stesso e lo rende vulnerabile al comando - il «talismano della imbecillità cui è legato il dominio dei tiranni» - ed è parimenti in grado di trasformare il desiderio di potere in una base accettabile e contrattata di convivenza civile. Lideale illuministico del bonbeur, della politesse e della sociabilité si incontra qui con il concetto romantico in gestazione di Bildung e fa valere la massimizzazione del benessere complessivo, o il beneficio della cooperazione sociale, quale principio normativo della moralità politica. In Helvetius la nozione di virtù, dopo essersi dissolta in La Mettrie e in Mandeville, rinasce dunque sotto nuove spoglie e sembra richiedere anche un nuovo tipo umano, gaio, agiato, attento ai propri diritti: «la gaiezza presuppone l'agiatezza e il segno dell'agiatezza di un popolo è ciò che certuni chiamano la sua insolenza, cioè la conoscenza che un popolo ha dei diritti dell'umanità, e di ciò che l'uomo deve all'uomo; conoscenza sempre interdetta alla povertà, timida e scoraggiata. L'agiatezza difende i suoi diritti, l'indigenza li cede» ${ }^{36}$. Morale, diritto, giustizia distributiva parlano con la medesima voce.

GIULIO PANIZZA

(31) Helvetius, De l'esprit (tr. it. cit., p. 77).

(32) Ivi.

(33) Ivi, p. 72.

(34) Ivi, p. 73.
(35) Montaigne, Essais, 1580 (tr. it di F. GaraviNI, Saggi, Milano, Adelphi, 1982, pp. 150-151).

(36) Helvetius, De l'esprit (tr. it. cit., p. 164). 